\title{
Psychosocial Competencies, Self-Efficacy and Performance of Nurses: A Comparative Study
}

\author{
Urvashi Singh $^{1 *}$, Shalini Singh ${ }^{2}$, Poonam ${ }^{3}$
}

\section{ABSTRACT}

A nurse is a healthcare professional who focuses on autonomous and collaborative care of individuals of all ages, families, groups and communities, sick or well and in all settings. Though nursing is both an art and science but it requires psychosocial competencies in staff members i.e. proper decision making, critical thinking, coping with emotions, interpersonal relationships and effective communication. This construct abreast with competencies plays an important role in enhancing their Self-Efficacy i.e. positive evaluation of oneself. When nurses have higher selfevaluation, it also leads to their better performance in this profession. But is this issue same in private and government hospitals? Taking this perspective in mind, the present study was designed to make a comparative study of psychosocial competencies, self-efficacy and performance in nurses. A sample of 100 head nurses (50 from government and 50 from private hospitals) belonging to an age group of 40-45 years having at least 5 year job experience of same set up was selected. Psychosocial Competence Scale (Anita \& Vijayalaxmi, 2007), Self-Efficacy Scale (Raif \& Jerusalem, 1995) and Nursing Performance Scale (Ward \& Felter, 1979) were administered to measure these variables. The results revealed that private hospital nurses scored high on psychosocial competencies, i.e. effective communication, decision making, empathy and critical thinking and also had better self-efficacy and performance as well than government hospital nurses.

Keywords: Nursing, Critical thinking, Self-Efficacy, Psychosocial competence and Performance

Nursing is the provision of care for individuals, families and groups throughout the entire lifespan - from conception to death. Nursing is both an art and a science that requires the understanding and application of the knowledge and skills specific to the discipline. It also draws attention on knowledge and techniques derived from the humanities and the physical social, medical and biological sciences.

\footnotetext{
${ }^{1}$ Asstt. Professor, Draunacharya College, Gurgaon, India

${ }^{2}$ Deptt. of Psychology, M.D. University, Rohtak, India

${ }^{3}$ Research Scholar, Deptt. of Psychology, M.D. University, Rohtak, India

*Responding Author

(c) 2016 I U Singh, S Singh, Poonam; licensee IJIP. This is an Open Access Research distributed under the terms of the Creative Commons Attribution License (http://creativecommons.org/licenses/by/2.0), which permits unrestricted use, distribution, and reproduction in any Medium, provided the original work is properly cited.
} 
World Health Organization (2000) defined that "The mission of nursing in society is to help individuals, families and groups to determine and achieve their physical, mental and social potential, and to do so within the challenging context of the environment in which they live and work. This requires nurses to develop and perform functions that relate to the promotion and maintenance of health as well as to the prevention of ill health. Nursing also includes the planning and implementation of care during illness and rehabilitation, and encompasses the physical, mental and social aspects of life as they affect health, illness, disability and dying.

Nursing errands have been classified into three categories: direct patient consideration, indirect patient consideration, and non-nursing assignments or task unrelated to nursing (Hobgood, Villani \& Quattlebaum, 2005). These errands have direct measures of performance.

Though these errands have direct measures of performance but to maintain good rapport and further dealing with the patients during and after treatment involve their psychosocial competence. WHO (1997) has defined it as 'Person's ability to deal effectively with the demands and challenges of everyday life. It is a person's ability to maintain state of mental well being and to demonstrate positive and adaptive approach in dealing and interacting with others in his/her culture and environment. Positive approach implies that a person is forward looking and even in different situations can find a ray of hope and opportunities to find solutions. Competency is more than just knowledge and skills. It involves the ability to meet complex demands by drawing on and mobilising psychosocial resources (Skills \& Attitudes) in a particular context.

Psychosocial competency plays a vital role in the positive attributes of an individual and selfefficacy is one of them. Self-efficacy is characterized as, "individuals' convictions about their capacities to deliver assigned levels of execution that practice impact over occasions that influence their lives. Self-efficacy convictions decide how individuals feel, think, persuade themselves and carry on. A strong sense of efficacy upgrades human achievement and individual prosperity from numerous points of view. Individuals with high certification in their capacities approach take difficult tasks as challenges to be mastered rather than as threats to be avoided. Such a viable viewpoint encourages natural intrigue and profound engagement in exercises. They set themselves testing objectives and keep up solid responsibility to them. They uplift and support their endeavors notwithstanding disappointment. They rapidly recuperate their feeling of adequacy after disappointments or setbacks. They ascribe inability to inadequate exertion or lacking information and aptitudes which are acquirable. They approach undermining circumstances with confirmation that they can practice control over them. Such an effective standpoint produces individual achievements, decreases stretch and lowers vulnerability to depression.”

Bandura (1992) stated that there are four wellsprings of self-efficacy i.e. Mastery experiences, Social modeling, Social persuasion and Psychological responses. All have their significant importance on the performance of an employee. The term 'performance' means output. In case 
of nurses it refers to the convenience framework in terms of patients treatment, autonomy, effective decision making, proper working conditions etc.

Forbes, Bott and Taunton (1997) found the positive relationship between self-efficacy, job autonomy and job satisfaction of nurses. Sagie (1994), Relf (1995) reported the relationship between dynamic working conditions, autonomy, functional role factors in nurses on their performance. The review of literature shows lot of work done on nurses in the context of positive psychological capital, burnout, stress etc. but there is a dearth of empirical evidence in relation to the psychosocial competency, self-efficacy and performance in nursing profession.

Moreover, in current scenario, there is vast growth of private hospitals where medical and paramedical staff is working. Whether these variables do have their variation in government and private hospitals nurses is a major objective of this investigation. With this impetus in mind, the present study is an attempt to make a comparative analysis of psychosocial competencies, selfefficacy and performance in government and private hospital nurses.

\section{METHOD}

Sample :

The present study was carried out on a sample of 100 nurses. 50 were taken from government and civil hospitals and 50 were working in private multispecialty hospitals. Al belonged to an age group of 40-45 years, were head nurses in hierarchy having at least 5-7 year experience working in same set-up. The sample was taken from various hospitals of Gurgaon and Faridabad Distt.

\section{Design :}

It is a two group design study where both the groups were compared on the variables of psychosocial competency, self-efficacy and performance.

\section{Tools :}

Psychosocial Competence Scale (Anita \& Vijayalaxmi, 2007): It deals with 10 life skills namely, Problem solving, decision making, critical thinking, creative thinking, empathy, selfawareness, coping with emotions, coping with stress, interpersonal relationships and effective communication. Each life skill is measured with 10 items having a total of 100 items on a 5 point likert scale. The low score indicates high competency and vice-versa.

Generalized Self-Efficacy Scale (Raif \& Jerusalem, 1995) : It has 10 items having 4 alternatives. The maximum score is 40 and minimum is 10 . The higher the score, the higher is self-efficacy.

Nursing Performance Scale (Ward \& Felter, 1979) : It is a self-regulated instrument having 52 items on a point scale assessing nursing performance. It deals with 6 execution subscales, i.e., leadership, critical care, teaching/collaboration, planning/evaluation interpersonal relations and professional development. The higher the score, the higher is the performance. 


\section{Procedure :}

After rapport establishment, all the tests were administered individually on each subject. The subjects were assured that their responses would be kept confidential. After data collection, the scoring was done as per the manual's guidelines. The guidelines of this type of investigation must be communicated to authorities of health care industry so that overall wellness of not only patients but also of nurses also get boost up.

\section{RESULTS AND DISCUSSION}

The objective of the present study was to assess and compare the psychosocial competencies, self-efficacy and performance in the nurses of government and private hospitals.

Table I : Mean, SD and t-value of Private and Government Hospital nurses on psychosocial competency, self-efficacy and performance

\begin{tabular}{|c|c|c|c|c|c|}
\hline \multirow[t]{2}{*}{ Variables } & \multicolumn{2}{|c|}{ Groups Means } & \multicolumn{2}{|c|}{ Groups SD's } & \multirow[t]{2}{*}{ t-values } \\
\hline & Private & Govt. & Private & Govt. & \\
\hline \multicolumn{6}{|c|}{ Psychosocial competency } \\
\hline Problem Solving & 22.70 & 26.75 & 5.44 & 4.22 & $3.82 * *$ \\
\hline Decision Making & 20.19 & 24.76 & 5.10 & 4.82 & $3.46^{* *}$ \\
\hline Critical Thinking & 22.82 & 25.86 & 4.49 & 5.68 & $3.24 * *$ \\
\hline Creative Thinking & 27.17 & 29.80 & 5.79 & 5.97 & $2.68 * *$ \\
\hline Empathy & 25.10 & 20.82 & 5.20 & 5.38 & $2.63 * *$ \\
\hline Self-Awareness & 27.17 & 25.12 & 5.12 & 5.29 & $3.12 * *$ \\
\hline $\begin{array}{l}\text { Coping with } \\
\text { Emotions }\end{array}$ & 25.67 & 27.82 & 4.13 & 5.18 & $3.62 * *$ \\
\hline Coping with Stress & 22.34 & 25.32 & 2.12 & 4.16 & $3.32 * *$ \\
\hline $\begin{array}{l}\text { Interpersonal } \\
\text { Relations }\end{array}$ & 23.16 & 25.12 & 4.37 & 6.08 & $4.16^{* *}$ \\
\hline $\begin{array}{l}\text { Effective } \\
\text { Communication }\end{array}$ & 23.12 & 26.18 & 4.42 & 5.12 & $2.56 * *$ \\
\hline Overall & 242.16 & 259.12 & 38.08 & 42.12 & $5.52 *$ \\
\hline GSE & 30.47 & 25.48 & 2.47 & 3.64 & $2.68 *$ \\
\hline Performance & 180.94 & 173.64 & 8.17 & 9.02 & $3.62 *$ \\
\hline
\end{tabular}

* 0.01 level of significance

** 0.05 level of significance

GSE $=$ Generalized self-efficacy

Table no. 1 clearly shows that the private hospital nurses had higher problem solving skill (22.70) than government nurses (26.75) and they have been found statistically significant. As far as other dimensions of psychosocial competencies are concerned, the private hospital nurses are statistically higher than government set-up nurses, i.e., in case of effective decision making, critical and creative thinking, coping with stressful and adverse situations, effective patient 
handling and maintaining good relations with the patients etc. Rash and Anderson (2009) found that communication skills in nurses plays an effective role in providing psychological support to patients, confidence in providing social support and their self-efficacy. Langewitz (2010) reported that post treatment effective communication skills in nurses acts as a cushion in patients' early and quick recovery. Towers (2007) found that nurses in private or multispecialty hospitals are more competent and effectively groomed in terms of patient care. Moreover working in private set-up, they take quick actions so that their public image of hospital remains high.

The obtained results also clearly reveal the statistically significant difference in private and government nurses in measures of self-efficacy, i.e. 30.47 and 25.48 and performance i.e. 180.94 and 173.64 respectively. Wilkinson (2008) and Remshardt (2012) studied nurses as giving post care to heart patients after bypass surgery. They found that nurses having more confidence, empathy and high in coping stressful situations could provide psychosocial support to patients which increased their wellness.

The obtained results clearly revealed that nurses working in private hospitals are better equipped in handling patients in terms of providing them psychosocial support and solace. At the same time, this study also clearly showed that the more psychosocial competence on the part of nursing staff is responsible for their own positive thinking, positive self-evaluation and overall performance. On the other hand, nurses working in government set-up may be having more job security and other benefits are less psychosocial competent and in performance. The attraction of general public towards private hospitals for their treatment and post care might be attributed to this. Therefore the need of an hour suggests to conduct time to time life skills training on psychosocial competencies so that nursing staff not only perform high but also increase their self-evaluation and of patients too.

\section{REFERENCES}

Anita, B. and Vijayalaxmi, A. (2007). Psychosocial Competence Scale in Adjustment, SelfEfficacy and Psychosocial Competency of Drug Addicted Adolecents (2013). Journal of Psychology, 4, 13-18.

Bandura, A. (1992). Exercise of Personal agency through the self-efficacy mechanisms. In R. Schwarzer (Ed.), Self-Efficacy: through control of action. Washington, DC: Hemisphere.

Forbes, S.A., Bott, M.J. and Taunton, R.L. (1997). Control over nursing practice: A construct coming of age. Journal of Nursing Measurement, 5, 179-190.

Hobgood, C., Villani, J. and Quattlebaum, R. (2005). Impact of emergency department volume on registered nurse time at the bedside. Annals of Emergency Medicine, 46, 481-489.

Langewitz, W. (2010). Swiss cancer league communication skills training programme for oncology nurses: an evaluation. Journal of Advanced Nursing, 66, 2266-2277. 
Raif, S. and Jerusalem, M. (1995). Generalized Self-Efficacy Scale. In J. Weinman, S. Wright and M. Johnston. Measures in Health Psychology: A User's portfolio. Causal and Control Beliefs (pp. 35-37). Windsor: UK.

Rask, M.T. and Anderson, M.L. (2009). Effects of an Intervention aimed at improving nurse patient communication in an oncology patient clinic. Cancer Nursing, 32, E1-E11.

Remshardt, M.A. (2012). Do you know your professional boundaries? Retrieved from http://journals.iww.com/nursingmadeincredibly easy.

Sagie, A. (1994). Participative decision making and performance: A moderator analysis. Journal of Applied Behavioural Science, 30, 227-246.

Ward, M.J. and Fetler, M.E. (1979). Instrument for use in nursing Education Research, Western Interstate Communication for Higher Education, Boulder, Colorad.

WHO (1997). World Health Organization. Life Skills Education for Children and Adolescents in Schools. Programme on Mental Health Organization, Geneva : WHO.

Wilkinson, S. (2008). Communication Skills Training for nurses working with heart disease. British Journal of Cardiac Nursing, 3, 475-481.

How to cite this article: U Singh, S Singh, Poonam (2016), Psychosocial Competencies, SelfEfficacy and Performance of Nurses : A Comparative Study, International Journal of Indian Psychology, Volume 3, Issue 3, No. 8, DIP: 18.01.150/20160303, ISBN: 978-1-365-12176-0 